\title{
The Inhibitory Ability of Cocoa Pod Husk Extract on Enterococcus faecalis Glucosyltransferase Enzyme Activity
}

\author{
Tamara Yuanita ${ }^{1}$, Ridzki A Oktavianti ${ }^{2}$, Debby F Suryani ${ }^{3}$, Mandojo Rukmo ${ }^{4}$, Sri Kunarti ${ }^{5}$, Andrie H Kusuma ${ }^{6}$
}

\begin{abstract}
Background: The presence of Enterococcus faecalis in root canal is considered as one of the factors causing root canal treatment failure since the bacteria are capable of producing glucosyltransferase enzymes that play a role in forming endodontic biofilms. Hence, the bacteria are resistant to antibiotics. On the other hand, cocoa pod husk extract which is rich in chemical components especially flavonoids, tannins, and saponins, is thought to have an ability to inhibit Enterococcus faecalis glucosyltransferase enzyme activity.

Aim: The aim of this research is to analyze the inhibitory ability of cocoa pod husk extract against $E$. faecalis glucosyltransferase enzyme activity. Materials and methods: A total of 27 research samples were divided into three groups, namely, positive control (chlorhexidine gluconate $2 \%)$, negative control (aquades), and cocoa pod husk extract 3.12\%. Next the enzymatic activity of each sample group was calculated based on the size of the fructose area read by high-performance liquid chromatography (HPLC) expressed in percent (\%) and then converted to $\mu \mathrm{mol} / \mathrm{mL}$ fructose which was considered as 1 unit of glucosyltransferase enzyme activity. Subsequently, the data were analyzed statistically using Kruskal-Wallis test.

Results: The results of data analysis using the Kruskal-Wallis test showed significant differences between groups of samples $(p<0.05)$.

Conclusion: Cocoa pod husk extract of 3.12\% has inhibitory effect on E. faecalis glucosyltransferase enzyme activity.

Clinical significance: The use of cocoa pod husk extract meets the requirements and is proven useful as an irrigation agent in the treatment of root canals, because it contains antibacterial properties against $E$. faecalis.

Keywords: Cocoa pod husk extract, Endodontic biofilm formation, Enterococcus faecalis, Glucosyltransferase enzyme, High-performance liquid chromatography.

The Journal of Contemporary Dental Practice (2020): 10.5005/jp-journals-10024-2786
\end{abstract}

\section{INTRODUCTION}

Root canal treatment failure is influenced by several factors. A study states that the factors generally causing failure of root canal treatment are the bacterial persistence, inadequate root canal obturation, untreated major and accessory canals, overextension of root filling material, the occurrence of complications during instrumentation (ledge or broken instruments in the root canal), etc. ${ }^{1}$ The bacterial persistence in the root canal is convinced as the main factor of root canal treatment failure. ${ }^{2}$

Bacteria that are often attributed to root canal treatment failure are E. faecalis. Enterococcus faecalis are facultative anaerobic bacteria that have an ability to form biofilms. Biofilm is a collection of microorganisms embedded in an extracellular polymeric substances (EPS) matrix and attached to a surface. ${ }^{3}$ The formation of these biofilms causes the bacteria to become more resistant to phagocytosis process, so the elimination of bacteria will be more difficult. ${ }^{4}$

The formation of $E$. faecalis biofilms, moreover, is influenced by several factors, one of which is glucosyltransferase enzyme activity. Glucosyltransferase is an enzyme that plays a role in hydrolyzing sucrose to fructose and glucose as well as in forming glucan. Glucan is an adhesin material playing a role in the process of biofilm formation. ${ }^{5}$

One method that can be used to eliminate this bacteria is by inhibiting glucosyltransferase enzyme activity. This inhibition of $E$. faecalis glucosyltransferase activity will then decrease glucan formation, resulting in reduced biofilm formation of $E$. faecalis. This condition subsequently triggers a decrease in the virulence of these bacteria. ${ }^{6}$
1,2,4-6 Department of Conservative Dentistry, Faculty of Dentistry, Universitas Airlangga, Surabaya, Indonesia

${ }^{3}$ Department of Conservative Dentistry, Universitas Airlangga, Surabaya, Indonesia

Corresponding Author: Tamara Yuanita, Department of Conservative Dentistry, Faculty of Dentistry, Universitas Airlangga, Surabaya, Indonesia, Phone:+62 8155130747, e-mail: tamara-y@fkg.unair.ac.id

How to cite this article: Yuanita T, Oktavianti RA, Suryani DF, et al. The Inhibitory Ability of Cocoa Pod Husk Extract on Enterococcus faecalis Glucosyltransferase Enzyme Activity. J Contemp Dent Pract 2020;21(3):271-276.

Source of support: Nil

Conflict of interest: None

The strategy recently used to eliminate bacteria in the root canal system is irrigation materials. The use of irrigation materials is expected to eliminate bacteria that exist in root canals especially those that cannot be reached with mechanical instrumentation.

Sodium hypochlorite and chlorhexidine gluconate are the irrigation materials commonly used during root canal treatment procedures. The use of these two ingredients is proven to have strong antibacterial activity; however, the use of these materials also has side effects, such as tooth discoloration, mucosal irritation, and strong odor., ${ }^{7,8}$

In addition, as another complication, the use of sodium hypochlorite which penetrates into the apical area can cause hematoma, ecchymosis, burning sensation, anesthesia paresthesia, etc. ${ }^{9,10}$ Due to those side effects, an alternative material that has

(c) The Author(s). 2020 Open Access This article is distributed under the terms of the Creative Commons Attribution 4.0 International License (https://creativecommons. org/licenses/by-nc/4.0/), which permits unrestricted use, distribution, and non-commercial reproduction in any medium, provided you give appropriate credit to the original author(s) and the source, provide a link to the Creative Commons license, and indicate if changes were made. The Creative Commons Public Domain Dedication waiver (http://creativecommons.org/publicdomain/zero/1.0/) applies to the data made available in this article, unless otherwise stated. 


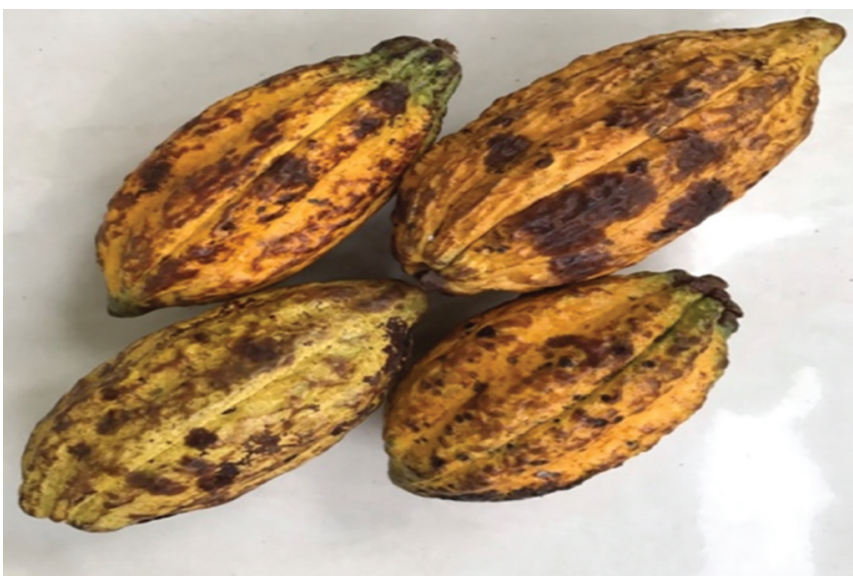

Fig. 1: Forestero chocolate fruit

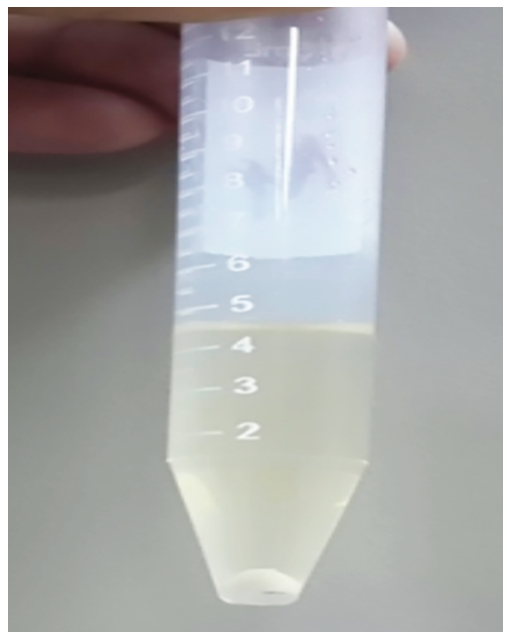

Fig. 3: Enterococcus faecalis supernatant containing glucosyltransferase enzyme

higher biocompatibility properties is required. Thus, the alternative material can be used safely (Figs 1 to 3 ).

Cocoa pod husk is a natural ingredient that can be processed as an alternative material to replace sodium hypochlorite and chlorhexidine gluconate. Phytochemical analysis on chocolate fruit peel extract shows many active compounds, such as alkaloid, tannin, saponin, terpenoid, and flavonoid that have antibacterial activity. ${ }^{11,12}$

Another study also shows that cocoa pod husk extract can inhibit the growth of Pseudomonas aeruginosa and Salmonella choleraesuis bacteria. ${ }^{13}$ Similarly, a study reveals that cocoa pod husk extract of $3.12 \%$ has minimum biofilm inhibitory concentration (MBIC) value against $E$. faecalis bacteria. ${ }^{14}$ Therefore, it can be said that cocoa pod husk has an antibiofilm effect on $E$. faecalis bacteria.

Unfortunately, many previous research only detected the number of biofilms formed using a spectrophotometer without regarding other factors that play a role in the process of biofilm formation, such as glucosyltransferase enzyme activity. As a result, further research is needed to be focused on the inhibitory ability of cocoa pod husk against $E$. faecalis glucosyltransferase enzyme activity playing an important role in biofilm formation. This research, consequently, aims to determine the inhibitory ability of cocoa pod husk extract with a concentration of $3.12 \%$ on E. faecalis glucosyltransferase enzyme activity.

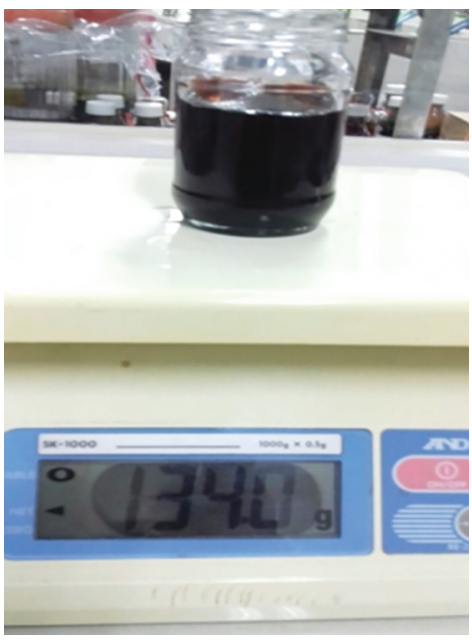

Fig. 2: Cocoa pod husk extract

\section{Materials and Methods}

This research is an experimental laboratory study with a posttestonly design. The number of samples in this research was 27 , which were divided into three groups, namely, positive control group using $2 \%$ chlorhexidine gluconate ( $2 \%$ chlorhexidine gluconate; Cercamed, Wola, Poland), negative control using sterile distilled water (Otsu Wl; Otsuka, Lawang, Indonesia), and treatment group using 3.12\% concentration of Forestero cocoa pod husk extract taken from plantations in Banyuwangi, East Java. This research involved three stages, namely, preparation of cocoa pod husk extract, purification of glucosyltransferase enzymes, and testing of glucosyltransferase enzyme activity (Figs 4 to 6).

In the first stage, cocoa pod husk extract preparation was performed at the Faculty of Pharmacy, Widya Mandala Catholic University, Surabaya. The extract was derived from cocoa pod husk by maceration technique using 70\% ethanol (Medis, Indonesia). First, $1 \mathrm{~kg}$ of chocolate fruit was dried for 5 days and then separated from the seeds. Second, cocoa pod husk was thinly cut and put into an oven (WTC Binder Oven FD 53; Tuttlingen, Germany) at a temperature of $50^{\circ} \mathrm{C}$ for 24 hours to become powder.

Ethanol of $70 \%$ was added until the powder was submerged in $1.5 \mathrm{~L}$ of liquid, and then it was tightly closed in a jar for 24 hours, shaken, and stirred. Evaporation was carried out using a water bath (Tyfsf DK-2000-IIIL; Huanghua, China) and filtered using filter paper (Whatman Filter Paper 1; Maidstone, United Kingdom) until a thick chocolate extract was obtained. The extract was then diluted to a concentration of $3.12 \%$.

In the next stage, purification of glucosyltransferase enzymes was carried out at the Research Center of Dentistry Faculty, Universitas Airlangga. First, local E. faecalis were inoculated in a culture media tube containing $10 \mathrm{~mL}$ of Brain Heart Infusion liquid (Oxoid CM1135 BHI; Thermo Scientific, Hampshire, United Kingdom) and then incubated at $37^{\circ} \mathrm{C}$ for 24 hours (Heratherm Incubator; Thermo Fisher Scientific, Waltham, United States). Second, the culture media tube was vibrated at $150 \mathrm{rpm}$ and centrifuged at 1,500 rpm for 30 minutes using a centrifuge (Medifuge Small Benchtop Centrifuge; Thermo Fisher Scientific; Waltham, United States) to obtain E. faecalis supernatant containing the glucosyltransferase enzyme (Tables 1 to 3 ).

Third, 27 test tubes were prepared and divided into three groups, namely, positive control group ( $2 \%$ chlorhexidine 


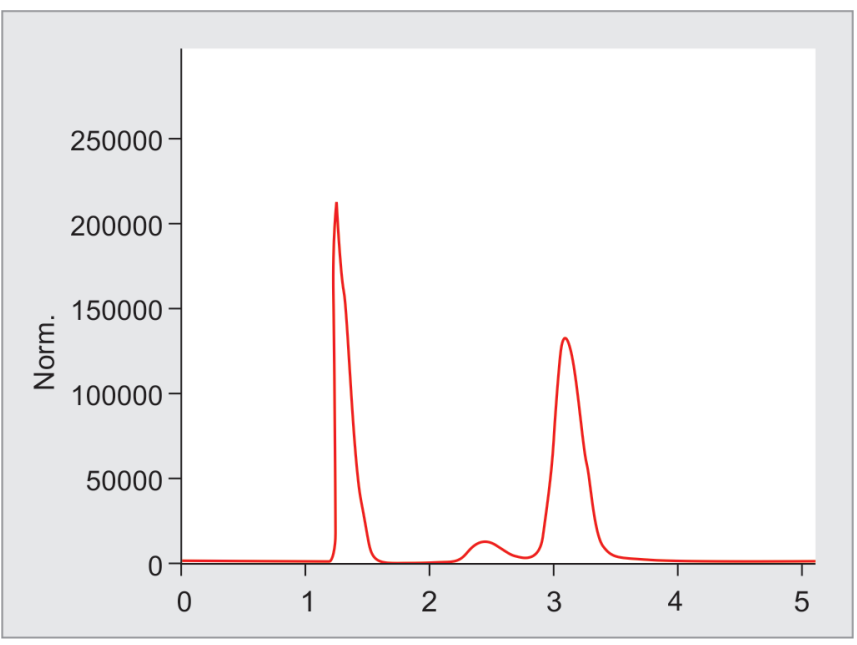

Fig. 4: Chromatogram of the chocolate fruit peel extract 3.12\%

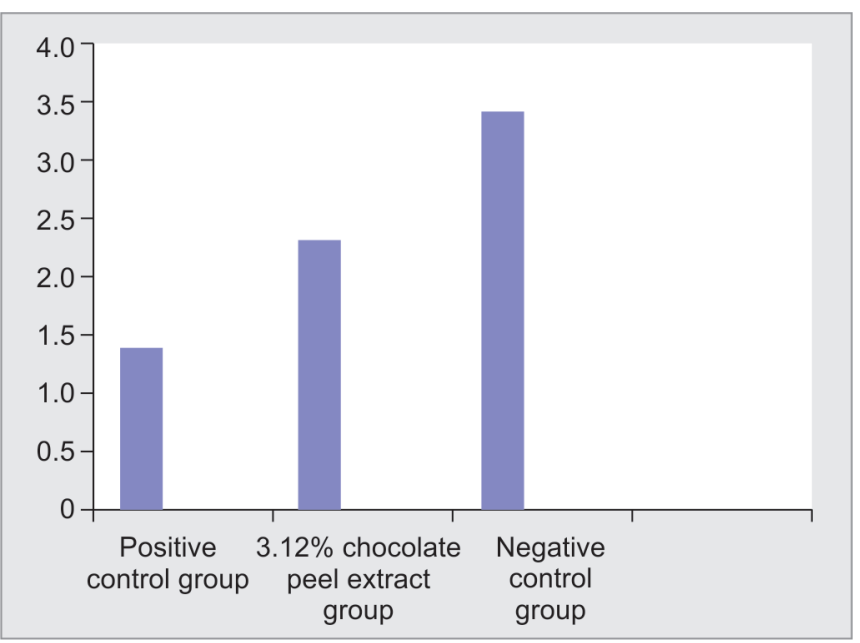

Fig. 6: Glucosyltransferase enzyme activity in each sample group

gluconate), negative control group (sterile distilled water), and treatment group added with $3.12 \%$ cocoa pod husk extract and a mixture of sucrose (Sigma Aldrich, Singapore), phosphate buffer (Sigma Aldrich, Singapore), and glucosyltransferase enzymes. The intervention in each group then was conducted by adding $0.9 \mathrm{~mL}$ sucrose $0.25 \mathrm{M}$ in a $\mathrm{pH} 7$ phosphate buffer, $0.1 \mathrm{~mL}$ of glucosyltransferase enzyme, and $0.025 \mathrm{~mL}$ of $2 \%$ chlorhexidine gluconate for the positive control group, sterile distilled water for the negative control group, or $3.12 \%$ cocoa pod husk extract for the treatment group. Subsequently $0.2 \mathrm{M}$ phosphate buffer with the $\mathrm{pH} 7$ was added to the total mixture volume of $2 \mathrm{~mL}$. All groups of samples were incubated at $37^{\circ} \mathrm{C}$ for 2 hours and then filtered.

And the last stage, testing of glucosyltransferase enzyme activity, was conducted at the Pharmacy Testing Service Unit of Airlangga University Surabaya. The activity of glucosyltransferase enzyme was tested by injecting as much as $2 \mu \mathrm{L}$ of the solution from each group on an high-performance liquid chromatography (HPLC) device (Agilent 1100 series; Waldbronn, Germany) and then observing the retention time. High-performance liquid chromatography is an analytical technique in chemistry used to separate, identify, and quantify each component in a mixture. Next the fructose level analyzed by chromatography was calculated by reading the fructose area based on the calibration curve formed

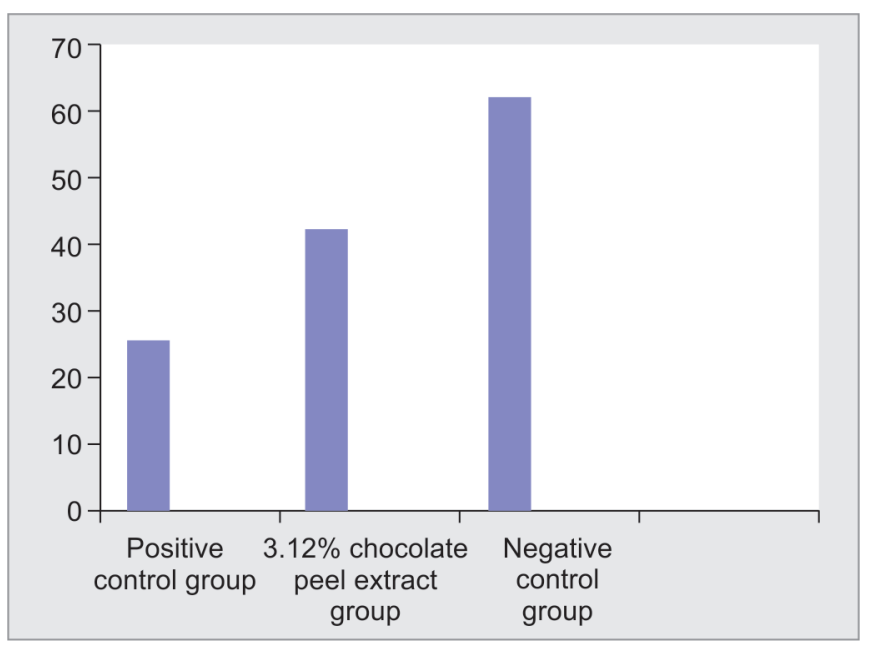

Fig. 5: Fructose concentrations (\%) in each sample

Table 1:The results of one-way ANOVA test on the fructose concentration

\begin{tabular}{ll}
\hline & Significance \\
\hline Difference among sample groups & 0.000 \\
\hline
\end{tabular}

Table 2: The results of Tukey HSD test on the fructose concentrations

\begin{tabular}{|c|c|c|c|}
\hline Sample groups & $\begin{array}{l}\text { Positive } \\
\text { control group }\end{array}$ & $\begin{array}{l}\text { Chocolate pod } \\
\text { husk extract } \\
3.12 \% \text { group }\end{array}$ & $\begin{array}{l}\text { Negative } \\
\text { controlgroup }\end{array}$ \\
\hline Positive control group & - & 0.000 & 0.000 \\
\hline $\begin{array}{l}\text { Chocolate peel } \\
\text { extract } 3.12 \% \text { group }\end{array}$ & 0.000 & - & 0.000 \\
\hline $\begin{array}{l}\text { Negative control } \\
\text { group }\end{array}$ & 0.000 & 0.000 & - \\
\hline
\end{tabular}

Table 3: The results of Kruskal-Wallis test on the glucosyltransferase enzyme activity

\begin{tabular}{ll}
\hline & Significance \\
\hline Difference among sample groups & 0.000 \\
\hline
\end{tabular}

from several concentrations of the standard fructose solution. The equation of the calibration curve in this study is as follows:

$$
Y=\mathrm{mx}+b
$$

$$
\begin{aligned}
& \text { Note: } \\
& Y=\text { Sample area } \\
& m=631134.17187 \\
& x=\text { Fructose concentration in sample (\%) } \\
& b=19546,51875
\end{aligned}
$$

A unit of glucosyltransferase enzyme activity is defined as $1 \mu \mathrm{mol}$ fructose $/ \mathrm{mL}$ derived from the enzyme/hour. An SPSS version 20 was performed in this research.

\section{Results}

Based on the results of the research conducted on 27 samples divided into three groups (positive control, negative control, and cocoa pod husk extract 3.12\%) using HPLC devices, the fructose concentration formed in each group was identified. The average 
fructose concentration in each group was $24.99 \%$ for the positive control group, $41.57 \%$ for the $3.12 \%$ cocoa pod husk extract, and $62 \%$ for the negative control group.

The concentration of fructose in each group was then converted to $\mu \mathrm{mol} / \mathrm{mL}$ referring to 1 unit of glucosyltransferase enzyme activity. The results showed 1.39 units for the positive control group, 2.3 units for the $3.12 \%$ cocoa pod husk extract, and 3.44 units for the negative control group.

Next, the results of fructose concentration and glucosyltransferase enzyme activity from each group were tested for homogeneity and normality. The results of both tests then determined the type of data analysis used.

The homogeneity test of fructose concentration showed that the fructose concentration data had normal homogeneity and normality. Therefore, one-way analysis of variance (ANOVA) test was conducted and then continued with the Tukey HSD test.

The results of the one-way ANOVA test on the fructose concentrations revealed that the significance of the difference among sample groups was $0.000(p>0.05)$. This indicates a significant difference in the concentration of fructose among the sample groups.

The Tukey HSD test was then carried out to determine the differences in the fructose concentration between the two groups of samples. The Tukey HSD test on the fructose concentration showed that each pair of sample groups had a significance value of less than 0.05 ( $p \leq 0.05$ ). The significance value of fructose concentration in each group was 0.000 for the positive control group, 0.000 for the $3.12 \%$ cocoa pod husk extract, and 0.000 for the negative control group. This shows that each pair of sample groups had a significant difference and also that each pair of sample groups had a significant difference.

Next the normality and homogeneity testsonglucosyltransferase enzyme activity revealed that the data distribution was normal, but the data were not homogeneous. Thus, Kruskal-Wallis test was performed on the data of glucosyltransferase enzyme activity.

The results of the Kruskal-Wallis test on the glucosyltransferase enzyme activity showed that the significance of difference among the research groups was 0.000 . The significance value of fructose concentration in each group was 0.000 for the positive control group, 0.000 for the $3.12 \%$ cocoa pod husk extract, and 0.000 for the negative control group. This indicates that there was a significant difference in glucosyltransferase enzyme activity among the research groups.

\section{Discussion}

Repeated infections after root canal treatment procedures are a sign of root canal treatment failure. The main factor that causes the root canal treatment failure is the presence of bacteria., ${ }^{1,2}$

Enterococcus faecalis are pathogenic bacteria often associated with root canal treatment failure. Enterococcus faecalis bacteria are also known as facultative anaerobic gram-positive bacteria that can penetrate into the dentinal tubules. ${ }^{15}$

Moreover, E. faecalis bacteria have several virulence factors that can cause infection in the host. The virulence factors possessed by E. faecalis bacteria are aggregation substance, surface adhesins, sex pheromones, gelatinase, cytolysin, and so on.

Enterococcus faecalis bacteria can also survive when the environmental conditions are not favorable since they have an ability to form biofilms. A research states that bacteria with their biofilms will be 1,000 times more resistant to phagocytic activity, antibodies, and antibacterial materials than with their free form/ planktonic form. ${ }^{15}$ The ability of E. faecalis to form biofilms, thus, makes these bacteria very difficult to eliminate. ${ }^{16}$

Biofilm is a collection of microorganisms embedded in a matrix of EPS and attached to a surface. ${ }^{3}$ Biofilm formation process consists of several stages: (a) initial stage: planktonic form of bacteria attached on a surface, (b) the second stage: cell aggregation to form microcolony and increase attachment caused by the production of extracellular polymeric substances (EPS); therefore, the attachment become irreversible (and the opening of microorganism cell surface structure), (c) the third stage: three-dimensional formation and biofilm maturation, providing protection against host defense mechanisms and antibiotics, and (d) the fourth stage: detachment (bacteria left the biofilms itself) and dispersion of planktonic bacteria ready to colonize other surfaces. ${ }^{17}$

Furthermore, exopolysaccharide often found in bacterial biofilms is glucan. Glucan is one of exopolysaccharide types composed of several glucoses (D-glucose monomers) which will form a polymer. The chemical reaction of glucan synthesis is triggered by these bacteria, which will be accelerated by glucosyltransferase enzyme. The enzyme will then hydrolyze sucrose to fructose and glucose and play a role in glucan formation. ${ }^{5}$

The role of glucosyltransferase enzyme actually is very large in the process of biofilm formation. Hence, now many studies aim to inhibit glucosyltransferase enzyme activity in order to inhibit the formation of biofilms. The inhibition of biofilm formation will make bacteria more susceptible to phagocytic activity and antibodies from host cells and antibacterial ingredients.

Cocoa pod husk, on the contrary, is a natural ingredient that has been known to be able to inhibit the biofilm formation of E. faecalis bacteria. ${ }^{14}$ As a result, the aim of this research is to analyze the inhibitory ability of cocoa pod husk extract against $E$. faecalis glucosyltransferase enzyme activity. In this research, the catalytic activity of the glucosyltransferase enzyme was measured by counting the number of enzyme products formed (fructose) from each sample group at a certain time using an HPLC device.

The results of the activity of the glucosyltransferase enzyme in this research actually were fructose and glucan. Fructose is produced in a free form, while glucan is a bond between glucose compounds. Fructose compounds in the free form were analyzed by HPLC, so that the activity of the glucosyltransferase enzyme was measured by the amount of fructose concentration formed. The amount of fructose detected by HPLC then would illustrate the formation of more glucan as a product of the catalytic reaction of the glucosyltransferase enzyme.

Subsequently, fructose concentrations obtained were converted into glucosyltransferase enzyme activity. The activity of the glucosyltransferase enzyme is defined as 1 umol fructose/ $\mathrm{mL}$ from the enzyme/hour. Data on the results of fructose concentrations, consequently, will be directly proportional to the activity of the glucosyltransferase enzyme. The higher the fructose concentrations, the higher will be the glucosyltransferase enzyme activity in a group of samples.

Chlorhexidine gluconate of $2 \%$ was used as a positive control to benchmark the effectiveness of the antibacterial materials commonly used in standard root canal treatment procedures to inhibit glucosyltransferase enzyme activity, while negative control used distilled water. This aims to understand the activity of the glucosyltransferase enzyme under normal circumstances. Cocoa pod husk extract with a concentration of $3.12 \%$ was used in this research since the extract was proven to have an MBIC against E. faecalis. ${ }^{14}$ 
The results showed that the highest average fructose concentration was in the negative control group (62\%), followed by the $3.12 \%$ cocoa pod husk extract group (41.57\%) and the positive control group (24.99\%). This was proportional to the activity of the glucosyltransferase enzyme in each group. The highest glucosyltransferase enzyme activity was in the negative control group (3.44 units), followed by the cocoa pod husk extract $3.12 \%$ group (2.3 units) and the positive control group (1.39 units).

The negative control sample group had the highest average fructose concentration compared to the other two sample groups. The high fructose concentration indicates that the very high catalytic activity of the glucosyltransferase enzyme in hydrolyzing sucrose to fructose and glucan. It is proven statistically that the glucosyltransferase enzyme activity in the negative control sample group was the highest compared to the other sample groups. This situation is because the negative sample group was not given a treatment that could affect the activity of the glucosyltransferase enzyme, so that in the negative control group the activity of glucosyltransferase enzymes runs normally without intervention.

The positive control group using $2 \%$ chlorhexidine gluconate, on the contrary, had the lowest average fructose concentration compared to the negative control group and the $3.12 \%$ cocoa pod husk extract group. The low fructose concentration in the positive control group indicates that an inhibition of the catalytic activity of the glucosyltransferase enzyme. Based on the results of the statistical analysis, it was also found that the positive control group had the lowest glucosyltransferase enzyme activity.

The inhibition of glucosyltransferase enzyme activity in the positive control group is probably due to the bactericidal effect of chlorhexidine gluconate. The mechanism of the antibacterial effect of chlorhexidine gluconate is to damage the cell membrane. Positively charged chlorhexidine binds negatively charged phospholipids in the cell wall and causes rupture, which in turn leads to cytoplasmic lysis and results in cell death. ${ }^{18}$

The group of $3.12 \%$ cocoa pod husk extract had a lower fructose concentration than the negative control group but slightly higher than the positive control group. It means that cocoa pod husk extract with a concentration of $3.12 \%$ has an ability to inhibit the catalytic activity of the glucosyltransferase enzyme, though not as much as that in the positive control group. This was even proven statistically, which showed the activity of glucosyltransferase enzymes in the group of cocoa pod husk extract was lower than that in the negative control group but higher than that in the positive control group.

The inhibitory ability of glucosyltransferase enzyme activity by cocoa pod husk extract is due to the presence of active ingredients, such as tannins, flavonoids, and terpenoids. The types of terpenoid compounds believed to affect the activity of glucosyltransferase enzymes are ursolic acid and oleanolic acid. ${ }^{19}$ Ursolic acid and oleanolic acid inhibit the glucosyltransferase enzyme by directly acting on the enzyme without competing with the presence of the substrate.

Flavonoids in cocoa pod husk extract can inhibit the activity of glucosyltransferase enzymes because of the proanthocyanidin and flavone compounds contained. Proanthocyanidin can inhibit the activity of E. faecalis glucosyltransferase enzyme since the compound is able to inhibit organic acid production and biofilm formation by cariogenic bacteria. ${ }^{20}$ Proanthocyanidine effectively reduced the synthesis of insoluble polysaccharide and affected bacterial glycolysis. Proanthocyanidine diminished bacterial attachment by altering the surface characteristic and forced bacteria to remain in planktonic form in order to minimize biofilm formation. ${ }^{21}$

Besides, flavonoids will also inhibit the activity of glucosyltransferase enzymes because the structure of the compound has hydroxyl group and double-bond group in C2 and $\mathrm{C} 3$ bonds, which allow nucleophilic bonds in the amino acid chain of the glucosyltransferase enzyme. ${ }^{21}$ The nucleophilic bond in the glucosyltransferase enzyme is an inhibitor of glucosyltransferase enzyme which then inhibits the enzyme activity.

Tannin is also an active ingredient that can inhibit the activity of the $E$. faecalis glucosyltransferase enzyme. The presence of this active compound will then cause a change in the bonding capacity with metal ions. This change is considered to cause a decrease in the activity of glucosyltransferase enzyme. ${ }^{22}$

Based on the results of the Tukey HSD test, the fructose concentration in each pair of sample groups had a significance value of less than 0.05 ( $p \leq 0.05$ ). This indicates significant differences in the fructose concentration in each pair of sample groups. The negative control group had a significant difference when compared with the cocoa pod husk extract group and the positive control group. This means that the addition of $2 \%$ chlorhexidine gluconate and $3.12 \%$ cocoa peel extract can inhibit the activity of the glucosyltransferase enzyme characterized by decreased fructose concentration compared to when not given treatment.

Similarly, the $3.12 \%$ cocoa pod husk extract group also had a significant difference with a higher average fructose concentration than that of the positive control group. This indicates that the inhibitory ability of $3.12 \%$ chocolate fruit peel extract was lower than that in the positive control group. This is likely to occur because $3.12 \%$ is the MBIC of cocoa pod husk extract. The optimum concentration of cocoa pod husk extract is needed to have the same or even higher inhibitory ability of glucosyltransferase enzyme than that of the positive controls. Therefore, the optimum concentration range of chocolate fruit extracts needed is from less than $3.12 \%$ to $12.5 \%$.

\section{Conclusion}

Chocolate fruit extract at the concentration of 3.12\% has inhibitory effect on E. faecalis glucosyltransferase enzyme activity. Further research about inhibitory ability of cocoa pod husk extract on glucosyltransferase enzyme activity and inhibitory of biofilm formation (in vivo studies) is required.

\section{References}

1. Tsabassum S, Khan FR. Failure of endodontic treatment: the usual suspect. European J of Dent 2016;10(1):144-147. DOI: 10.4103/13057456.175682.

2. Pecora CN, Baskaradoss JK, Al-Sharif A, et al. Histological evaluation of root apices of failed endodontic cases. Saudi Endod J 2015;5(2): 120-124. DOI: 10.4103/1658-5984.155450.

3. Rosen E, Tsesis I, Elbahary S, et al. Eradication of Enterococcus faecalis biofilms on human dentin. Front Microbiol 2016;7:2055. Lewandowski Z, Bayenal H. Fundamental of Biofilm Research, 2nd ed., Broken South Park: CRC Press; 2014. p. 4.

4. Lee Y.Biofilm formation and antimicrobial resistance in enterococcus. Infect Chemother 2017;49(3):236-237. DOI: 10.3947/ic.2017.49.3.236.

5. Michiyo MN. Role of streptococcus mutans surface protein for biofilm formation. Jpn Dent Sci Rev 2017;54(1):22-29.

6. Rainey K, Michalek SM, Wen ZT, et al. Glycosyltransferase-mediated biofilm matrix dynamics and virulence of streptococcus mutans. Appl Environ Microbiol 2019;85(5):e02247-18. DOI: 10.1128/ AEM.02247-18. 
7. Zare Jahromi M, Tahmoorespoor A, Hemmat N, et al. The comparison of antibacterial effect of propolis, sodium hypochlorite $5.25 \%$, and chlorhexidine $2 \%$ as intracanal irrigants against Enterococcus faecalis: an ex vivo study. Caspian J Dent Res 2017;6(1):29-35.

8. Pribadi N, Yonas $Y$, Saraswati W. The inhibition of streptococcus mutans glucosyl transferase enzyme activity by mangosteen pericarp extract. Dent J (Majalah Kedokteran Gigi) 2017;50(2):97-101. DOI: 10.20473/j.djmkg.v50.i2.p97-101.

9. Mathew ST. Risks and management of sodium hypochlorite in endodontics. J Oral Hyg Health 2015(03):1-5. DOI: 10.4172/23320702.1000178.

10. Faras F, Abo-Alhassan F, Sadeq A, et al. Complication of improper management of sodium hypochlorite accident during root canal treatment. J Int Soc Prev Community Dent 2016;6(5):493-496. DOI: 10.4103/2231-0762.192939.

11. Nguyen VT, Nguyen NH. Proximate composition, extraction and purification of theobromine from cacao pod husk (Theobroma cacao L.). Technologies 2017;5(14):1-10. DOI: 10.3390/technologies5020014.

12. Mu'nisa A, Pagarra H, Maulana Z. Active compounds extraction of cocoa pod husk (Theobroma cacao I.) and potential as fungicides. In Journal of Physics: Conference Series, vol. 1028, Mo. 1. IOP Publishing; 2018. p. 012013.

13. Adi-Dako O, Ofori-Kwakye K, Frimpong Manso S, et al. Physicochemical and antimicrobial properties of cocoa pod husk pectin intended as a versatile pharmaceutical excipient and nutraceutical. J Pharm 2016;2016:1-12. DOI: 10.1155/2016/7608693.
14. Yuanita T, Putri DV, Rukmo M, et al. Antibiofilm power of cocoa bean pod husk extract (theobroma cacao) against Enterococcus faecalis bacteria (in vitro). IMEDITEC Johor Malaysia 2017.

15. Ashwini SC. Extreme resistance of Enterococcus faecalis and its role in endodontic treatment failure. Progress Med Sci 2018;2(1):9-13.

16. Chen $L, B u Q, X u H$, et al. The effect of berberine hydrochloride on Enterococcus faecalis biofilm formation and dispersion in vitro. Microbiol Res 2016: 186-187. DOI: 10.1016/j.micres.2016.03.003.

17. Jamal $\mathrm{M}$, Tasneem U, Hussain T, et al. Bacterial biofilm: its composition, formation and role in human infections. RRJMB 2015;4:1-14.

18. Renuka $S$, Muralidharan NP. Comparison in benefits of herbal mouthwashes with chlorhexidine mouthwash: a review. Asian J Pharm Clin Res 2017;10(2):3-7. DOI: 10.22159/ajpcr.2017.v10i2.13304.

19. Vesileva T, Bivolarski V, Bozov P, et al. Influence of ursolic acid on glucooligosaccharides synthesis by dextransucrase from leuconostocmesenteroides Lm 28. J Bio Scie Biotechnol 2015;4(2): 175-183.

20. Slobodníková L, Fialová S, Rendeková K, et al. Antibiofilm activity of plant polyphenols. Molecules 2016;21(12):1717. DOI: 10.3390/ molecules21121717.

21. Trentin DS, Silva DB, Frasson AP, et al. Natural green coating inhibits adhesion of clinically important bacteria. Sci Rep 2015;5(1):8287. DOI: 10.1038/srep08287.

22. Ren Z, Chen L, Li J, et al. Inhibition of streptococcus mutans polysachharide synthesis by molecules targeting glucosyltransferase activity. J Oral Microbiol 2016;8(1):1-9. DOI: 10.3402/jom.v8.31095. 